\title{
MC X-Ray, The Monte Carlo Program for Quantitative Electron Microscopy of Real Materials
}

\author{
P. Michaud and R. Gauvin \\ Department of Materials Engineering, McGill University, M. H. Wong Bldg, \\ 3610 University Street, Montréal, Québec, Canada, H3A 2B2.
}

MC X-Ray [1] is a new Monte Carlo program that allows quantitative electron microscopy of real materials. This program is an extension of the Monte Carlo programs Casino [2] and Win X-Ray [3] since it computes the complete $\mathrm{x}$-ray spectra from the simulation of electron scattering in solids of various types of geometries. This new program, which has been completely reprogrammed in $\mathrm{C}++$ under a window environment, is a real improvement because Win X-Ray is only able to compute x-ray spectra of homogeneous materials and CASINO performs only the computation of net x-ray intensities in a limited set of geometries. MC X-Ray allows more than 100 different regions in the materials having shape of spheres, cylinders and combinations of horizontal and vertical planes. All these regions can have a different composition.

As an example, simulated 128 X 128 images of a $20 \mathrm{~nm}$ thin foil of a 50 (wt.) \% B Fe alloy with a $20 \mathrm{~nm}$ square $\mathrm{W}$ phase (left) and a $20 \mathrm{~nm}$ square $\mathrm{Cr}$ phase (right) at $200 \mathrm{keV}$ with 50 e per pixels were performed. Figure [1] shows the B $K \alpha$ generated intensity map. The length of the $\mathrm{x}$ scan was twice the length of the $\mathrm{y}$ scan and this explains the rectangular shape of the $\mathrm{W}$ and $\mathrm{Cr}$ phases. Figure [2] shows the B $K \alpha$ emitted intensity map and absorption effects are seen towards the x-ray detector located towards the top of the image with a $20^{\circ}$ TOA. Figure [3] shows the Fe $K \alpha$ emitted intensity map and absorption effects are negligible because of its higher photon energy. Figure [4] shows a dark field image. Bright field images can also be simulated as well as High Annular Dark Field Images with a choice of collection angles from the user.

Details to obtain Mc X-Ray are given at http://montecarlomodeling.mcgill.ca/.

\section{References:}

1.P. Michaud and R. Gauvin (2009), "MC X-Ray, a New Monte Carlo Program for Quantitative X-Ray Microanalysis of Real Materials", Microscopy and Microanalysis, 15 (Supp.2), p. 488-489.

2.P. Hovington, D. Drouin and R. Gauvin (1997), "Casino: A New Era of Monte Carlo Code in C Language for Electron Beam Interaction, Part I: Description of the Program”, Scanning, Vol.19, pp. 1-14. 3.R. Gauvin, E. Lifshin, H. Demers, P. Horny and H. Campbell (2006), "Win X-ray, a new Monte Carlo Program that Computes X-ray Spectrum for X-ray Microanalysis in the Scanning Electron Microscope", Microscopy \& Microanalysis, 12, pp. 49 - 64. 


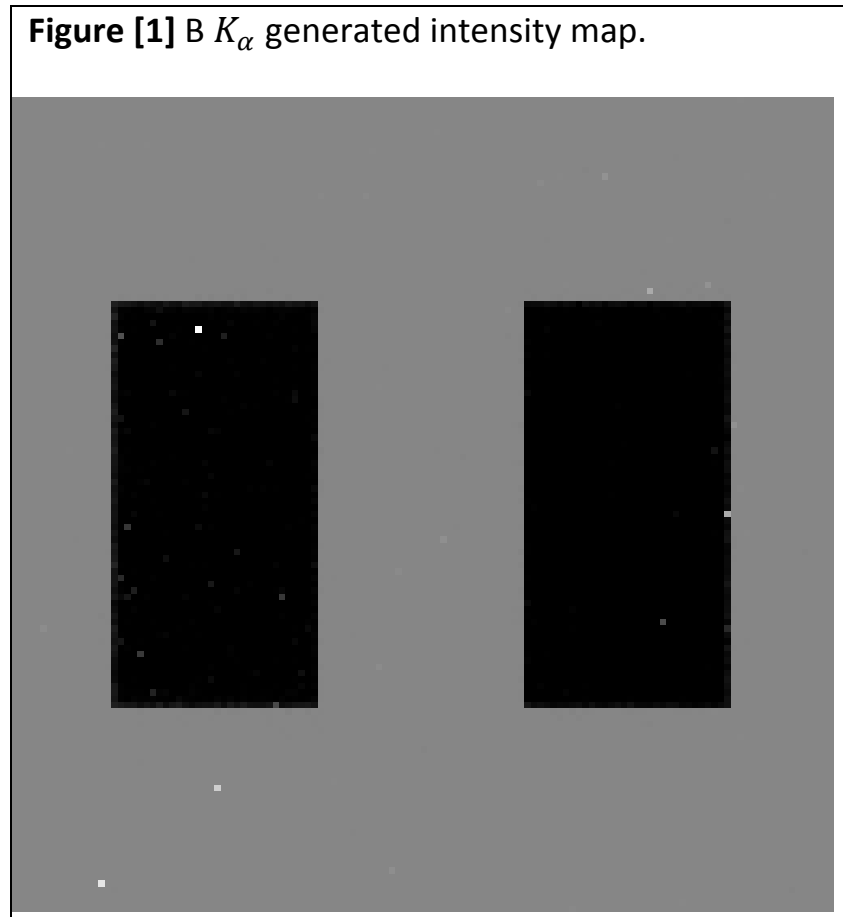

Figure [2] B $K_{\alpha}$ emitted intensity map.

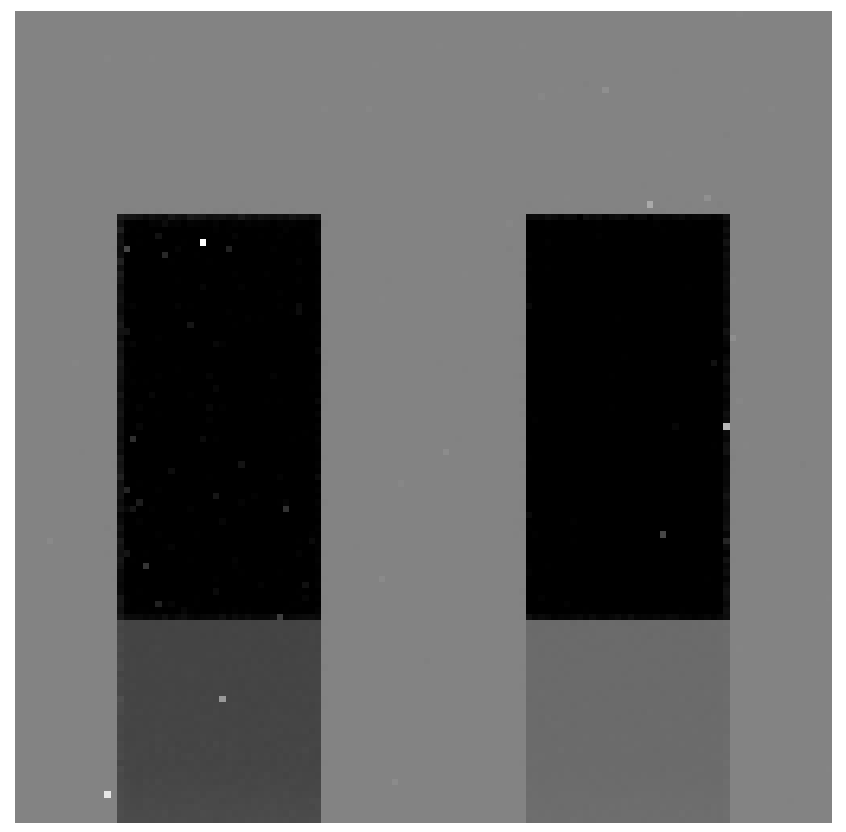

\section{Figure [3]}
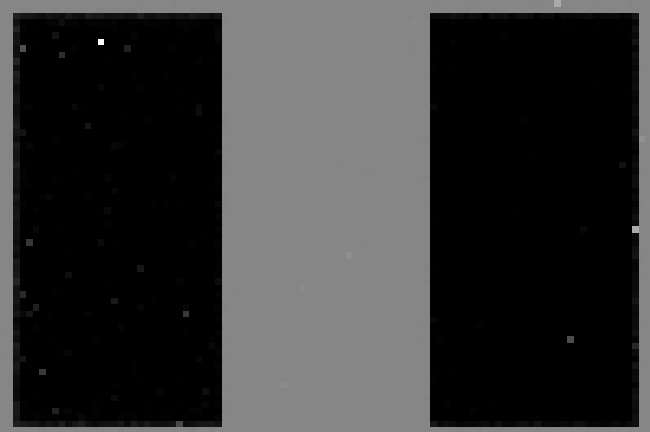

Fe $K_{\alpha}$ emitted intensity map.

Figure [4]

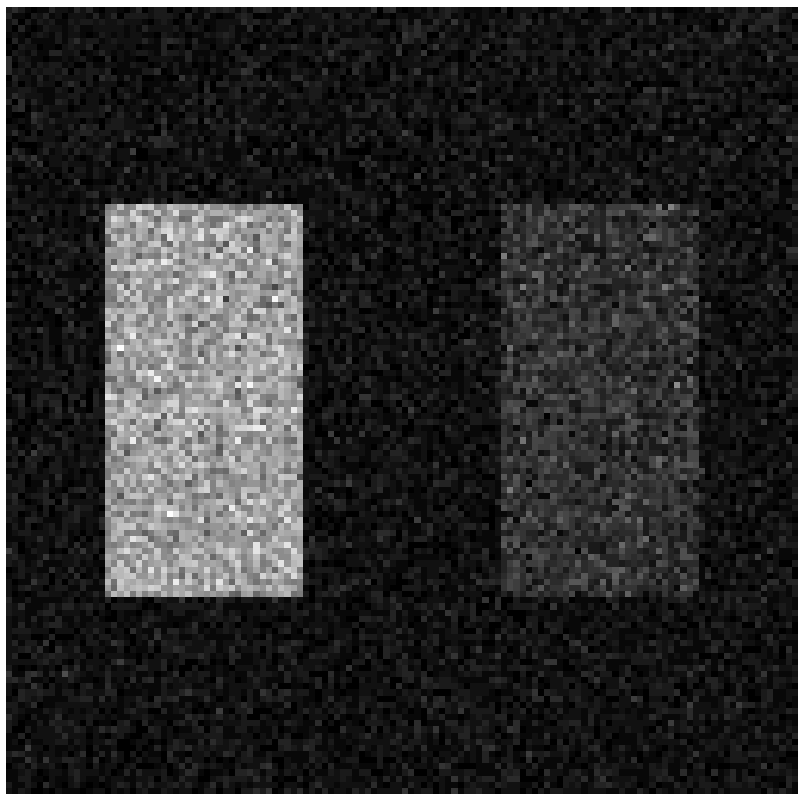

Dark Field image. 